\title{
The Helium Field Effect Transistor (II): Gated Transport of Surface-State Electrons Through Micro-constrictions
}

\author{
F. Shaban ${ }^{1}$ - M. Ashari ${ }^{1}$ - T. Lorenz ${ }^{1}$.

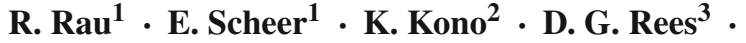 \\ P. Leiderer ${ }^{1}$
}

\begin{abstract}
We present transport measurements of surface-state electrons on liquid helium films in confined geometry. The measurements are taken using split-gate devices similar to a field effect transistor. The number of electrons passing between the source and drain areas of the device can be precisely controlled by changing the length of the voltage pulse applied to the gate electrode. We find evidence that the effective driving potential depends on electron-electron interactions, as well as the electric field applied to the substrate. Our measurements indicate that the mobility of electrons on helium films can be high and that microfabricated transistor devices allow electron manipulation on length scales close to the interelectron separation. Our experiment is an important step toward investigations of surface-state electron properties at much higher densities, for which the quantum melting of the system to a degenerate Fermi gas should be observed.
\end{abstract}

Keywords Helium field effect transistor · Surface electrons · Helium film · Electron transport · Confined geometry

\section{Introduction}

Electrons trapped above the surface of liquid helium form an ideal Coulomb system [1]. Because the electrons 'float' several $\mathrm{nm}$ above the liquid surface, interactions

\footnotetext{
P. Leiderer

Paul.Leiderer@uni-konstanz.de

1 Department of Physics, University of Konstanz, 78457 Constance, Germany

2 RIKEN, Hirosawa 2-1, Wako, Saitama, Tokyo 351-0198, Japan

3 NCTU, Hsinchu City 300, Taiwan
} 
between the charges and the helium substrate are generally weak, leading to the highest recorded electron mobilities [2,3]. On 'bulk' liquid substrates, the electron density is limited to a maximum value of $\sim 2 \times 10^{9} \mathrm{~cm}^{-2}$ due to an electrohydrodynamic instability of the helium surface. At such low densities, the electron system is essentially classical in nature, with strong Coulombic interaction between neighboring particles. This leads to the observation of many unique phenomena such as Wigner crystallization at low temperatures and anomalous magnetotransport due to many-electron effects [1]. However, it is of considerable interest to extend the range of densities investigated to higher values in order to observe the crossover from classical to quantum mechanical behavior. This can be achieved by using thin van der Waals helium films as a substrate. Electron densities of $\sim 10^{11} \mathrm{~cm}^{-2}$ have already been reached in this manner [4]. However, because the thickness of such highly charged helium films $(\sim 10 \mathrm{~nm})$ is comparable with the typical roughness of the underlying substrate, the mobility of electrons on thin helium films has been found to be typically orders of magnitude lower than in the 'bulk' case.

The exceptional purity of the system has led to proposals that surface-state electrons could be used in quantum information processing or quantum optics applications [5,6]. Motivated by these goals, researchers have recently developed microchannel devices, fabricated by multilayer lithography techniques, capable of manipulating single electrons, or single electron chains [7-9]. In microchannels of helium depth $\sim 1 \mu \mathrm{m}$, the electron system retains its bulk mobility. However, despite the reduced geometry, the maximum electron density is still limited by electrohydrodynamic instabilities and is increased only marginally over that of the bulk system [4]. Also, the fabrication of such devices is challenging, as the sample is typically composed of several metallic layers isolated from one another. In this study, we demonstrate that simple planar electrode devices, fabricated in only one lithographic step on silicon wafers, can allow the sensitive control of electron transport on thin-film substrates. Furthermore, we show that the transport measurements in such devices are not greatly limited by the decrease in electron mobility expected due to substrate roughness. Our experiments are an important step toward the study of electron transport in the 'quantum melting' regime that should occur at high electron densities.

So far, the transport experiments with surface-state electrons (SSE) in narrow channels between two reservoirs have been mostly carried out close to equilibrium, i.e., with nearly the same electron density in both reservoirs [7,10,11]. A small ac driving potential was coupled to one reservoir, and the signal resulting from the slightly oscillating electron density in the other reservoir was picked up capacitively using a lock-in technique. In the present study, however, we investigate the dynamics of the transport of SSE through the gate channel under conditions far off equilibrium. For this purpose, we use a source-gate-drain configuration, similar to a FET [12], and start with a situation where the source (S) is full and the drain (D) is empty. We open the channel completely, but only for a short time, then close it again, and measure the total amount of electrons arriving at the drain.

A crucial quantity for interpreting our data is the electron density in the source at the channel entrance. This problem was investigated previously with the same setup in Ref. [13]. There it was shown that due to the externally applied potential gradient along the sample, the electron density is not constant across the source area, but 
reaches its maximum value $n_{0}$ directly at the channel entrance. In Ref. [13], a model was developed which allows one to determine $n_{0}$ quantitatively from the potentials applied to $S, D$, and the gate $G$. The electron densities $n_{0}$ studied here were between $10^{6}$ and $10^{10} \mathrm{~cm}^{-2}$, that is in a range where the SSE behave like a classical system. A preliminary report of some of the results presented here has already been given in Ref. [14].

\section{Experimental Setup and Method}

The principle of the setup and the samples used in our experiments have been described in Ref. [13], and a sample chip with FET geometry as well as examples for gate channels (long, short, and structured) is shown in Fig. 1. A rectangular piece of a silicon wafer, covered with a 500 -nm-thick oxide, serves as a substrate for the S-G-D configuration formed by evaporated Au film structures. The typical roughness (RMS) of the silicon oxide, determined by atomic force microscopy, was $0.45 \mathrm{~nm}$ across an area of $5 \times 5 \mu \mathrm{m}^{2}$. Since we are using undoped silicon, the substrate is insulating at low temperature. Its conductivity can, however, be increased by external illumination, and under this condition, a voltage applied between the edges of S and D provides a well-defined linear potential gradient for the SSE [13]. A typical value for the potential

(a)

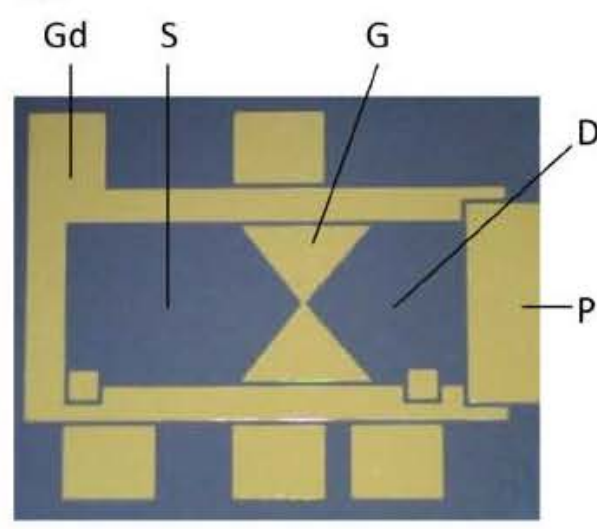

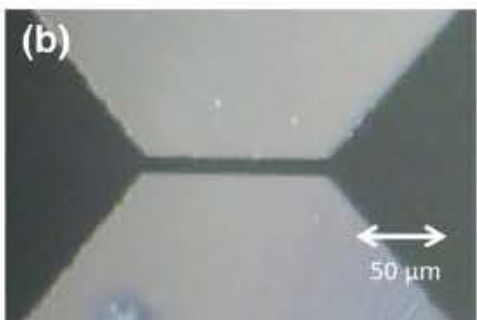
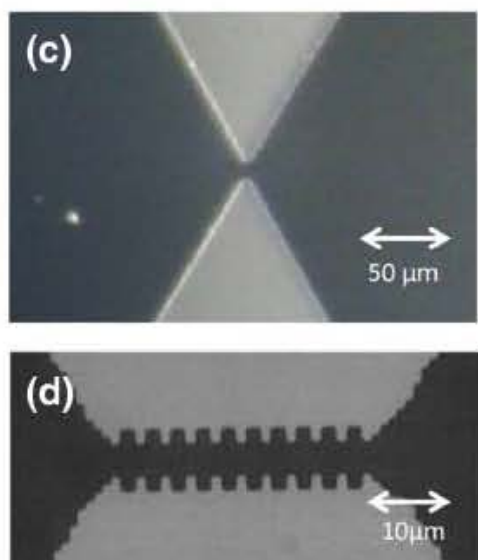

Fig. 1 Examples of a typical sample chip $(22 \mathrm{~mm} \times 18 \mathrm{~mm})$ with $S$ source, $G$ split gate, $D$ drain, $P$ pickup, $G d$ guard; b a long gate channel $(10 \mu \mathrm{m}$ wide); $\mathbf{c}$ a short gate channel $(10 \mu \mathrm{m}$ wide); and $\mathbf{d}$ a structured gate channel (3.75 and $7.7 \mu \mathrm{m}$ wide, respectively) (Color figure online) 


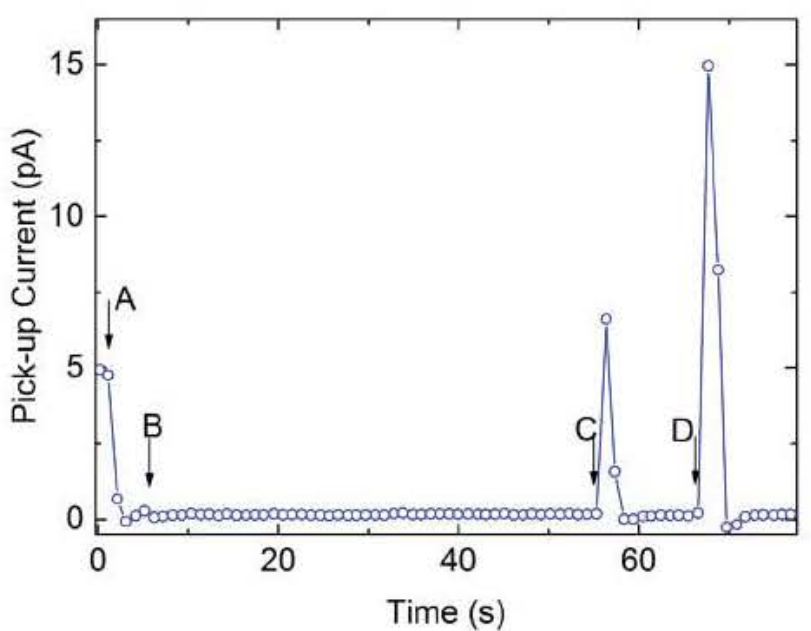

Fig. 2 Pickup current $I_{\mathrm{p}}$ as a function of time, representing a typical sequence for taking the data points shown in Figs. 3, 4 and 5. For details, see text (Color figure online)

difference between $S$ and $D$ is $1 \mathrm{~V}$, and the gate voltage is also in the range of a few $\mathrm{V}$. The electrons used to charge the source are supplied by a tiny filament, which is periodically heated with short current pulses at a repetition rate of $4 \mathrm{~Hz}$. As the gate channel is opened, electrons pass from $\mathrm{S}$ to $\mathrm{D}$ and are collected by a pickup electrode in the drain area. The typical dimensions of the gate channels used here range from 3.5 to $20 \mu \mathrm{m}$ in width and from 10 to $400 \mu \mathrm{m}$ in length. In an experimental run, the sample was kept at a fixed height of $\sim 1 \mathrm{~mm}$ above the bulk liquid ${ }^{4} \mathrm{He}$ level and was thus covered by a He film with a thickness of $\sim 60 \mathrm{~nm}$ in the flat portions of the sample. In the channel, the film thickness is expected to be somewhat larger due to capillary forces exerted by the 100 -nm high channel walls. The temperature in all the runs shown here was $1.3 \mathrm{~K}$; hence, the films were superfluid.

A typical time sequence for taking a data point is presented in Fig. 2. Shown is the pickup current $I_{\mathrm{p}}$ measured at the drain when the gate voltage is manipulated. In the beginning, the filament which supplies the electrons is on and the gate is open. The dc pickup current registered for that particular run was $5 \mathrm{pA}$. Then, the gate was closed at A, $I_{\mathrm{p}}$ dropped to zero, and the electrons from the filament filled the source. After a few seconds, a high enough charge density was reached that electrons started to spill over the gate barrier [13], and the filament was switched off at B. Under this condition, the electrons can be stored in S for times much longer than minutes. In the example given here, a gating pulse which opened the gate channel was applied for a short time after $55 \mathrm{~s}$ at $\mathrm{C}$. The electrometer registers the charge arriving at the pickup electrode as a pulse which is much wider than the actual electron pulse moving through the gate channel because of the slow response of the electrometer (which due to its integration time of $1 \mathrm{~s}$ measures charges correctly, but not currents on a timescale $\ll 1 \mathrm{~s})$. After another $10 \mathrm{~s}$ (D) the gate channel was opened completely for an extended period, and all the remaining (mobile) electrons stored in the source were transported to the drain. The sum of the charges picked up in the two pulses represents the total amount $N_{\mathrm{s}}$ 
of mobile electrons present in $\mathrm{S}$ at the beginning of the pulse sequence. This quantity was therefore used to control that the conditions during a run did not change and, in particular, that the total number of mobile electrons remained more or less constant.

\section{Results}

\subsection{Single Pulses}

\subsubsection{Long Channels}

We start with a description of the results for single gating pulses and channels whose length is considerably larger than the width, as shown in Fig. 1b. An example for the number of electrons, $N$, transmitted through a $100-\mu \mathrm{m}$-long and $10-\mu \mathrm{m}$-wide channel as a function of pulse width $\Delta t_{\mathrm{p}}$ is shown in Fig. 3. The total number of electrons in the source was $N_{\mathrm{s}} \sim 2 \times 10^{8}$ in this case. Since this value was fluctuating somewhat from pulse to pulse, we have used $N_{\mathrm{s}}$ (determined as illustrated in Fig. 2 for each data point) to normalize the data and have plotted the resulting normalized number of transmitted electrons, $N / N_{\mathrm{s}}$. It is observed that for short pulses the transmitted charge rises linearly, as the pulse width is increased. Then the data bend over and saturate, which means that the charge in the source is depleted by the first pulse. In Fig. 3, the 'depletion time' for reaching this state is about $4 \mathrm{~ms}$.

Figure 3 looks like the discharge of a capacitor $C$ (source) via a resistor $R$ (channel), with a characteristic time constant $\tau=R C$. However, the situation here is quite different from the 'normal' discharge of a capacitor, where the density of the electrons carrying the current through $R$ (the conduction electrons) is constant during the discharge and the driving force acting on the electrons is proportional to the voltage at $C$

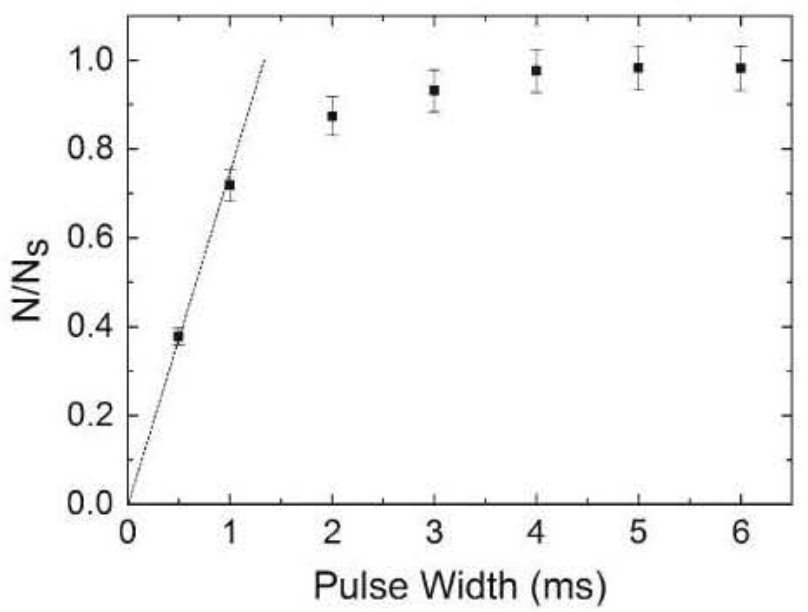

Fig. 3 Normalized number of electrons, $N / N_{\mathrm{s}}$, transmitted from source to drain, as the gate is opened by positive pulses of various widths $\Delta t_{\mathrm{p}}$. The channel in this sample was $100 \mu \mathrm{m}$ long and $10 \mu \mathrm{m}$ wide. The dashed line corresponds to a current of $\sim 1.5 \times 10^{11} \mathrm{e} / \mathrm{s}$ (from Ref. [14]) 


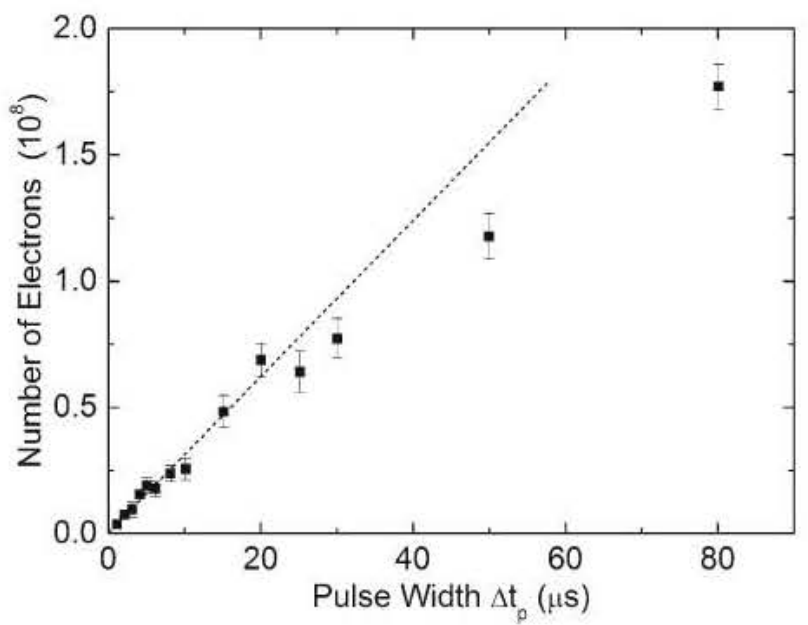

Fig. 4 Number of transmitted electrons per pulse versus pulse width $\Delta t_{\mathrm{p}}$ for a channel $100 \mu \mathrm{m}$ long and $20 \mu \mathrm{m}$ wide. The total number of electrons in the source was here $N_{\mathrm{s}}=2.5 \times 10^{8}$. The slope of the straight line corresponds to a current of $3.1 \times 10^{12} \mathrm{e} / \mathrm{s}$

(=the potential drop across the resistor), which decreases exponentially as the capacitor is discharged. In contrast, in our experiment the electron density at the channel entrance [which (roughly) is also the density of conduction electrons during transport] drops during the discharge of the source, and the external driving force, given by the potential gradient in the substrate, is constant during the discharge. To make the situation here even more involved, the relationship between the charge density at the channel entrance and the total charge in the source is nonlinear (see Ref. [13], Figs. 6,7). A further important aspect, which will be corroborated further below, is that the self-field of the charges in the source adds to the driving field. As a consequence, we will not analyze the whole discharge process here, but in the following concentrate on the linear part in the beginning. The initial slope in Fig. 3 corresponds to a current of $\sim 1.5 \times 10^{11} \mathrm{e} / \mathrm{s}$, or $24 \mathrm{nA}$, when the gate is opened.

Figure 4 presents data for a second sample, where the channel also was $100 \mu \mathrm{m}$ long, but a factor of 2 wider $(20 \mu \mathrm{m})$. Again, an initial linear portion is observed, but the slope was in this case distinctly steeper than in Fig. 3, namely $3.1 \times 10^{12} \mathrm{e} / \mathrm{s}$. The larger width of the channel can only partly explain the difference, meaning that the mobility of the electrons in the channel must have been higher in this run. A variation in mobility is not unusual for electrons on thin helium films; details of the substrate roughness and local potential fluctuations can play an important role $[15,16]$. Also, a variation of the helium film thickness, which was not very precisely controlled in our experiment, can have a major effect on the mobility [16-18].

From the initial current of $3.1 \times 10^{12} \mathrm{e} / \mathrm{s}$, one can obtain an estimate for the electron mobility, if one knows the electron density in the channel. Based on the model described in Ref. [13], the electron density $n_{0}$ at the channel entrance for the parameters of this measurement is calculated to be $9.8 \times 10^{8} \mathrm{~cm}^{-2}$. We assume for simplicity that the electron density $n$ in the channel is close to this value. The particle current 
through the channel can be expressed as

$$
I / e=w n \mu E
$$

where $I$ is the measured electrical current, $e$ is the electron charge, $w$ is the channel width, $\mu$ the mobility, and $E$ the electric field. For a channel width of $20 \mu \mathrm{m}$ and an externally applied electric field of $0.5 \mathrm{~V} / \mathrm{cm}$, this yields

$$
\mu=3.0 \times 10^{5} \mathrm{~cm}^{2} / \mathrm{Vs}
$$

This value is slightly higher than the one reported for the mobility on bulk ${ }^{4} \mathrm{He}$ at that temperature. One has to keep in mind, though, that this estimate is an upper limit, because the electric field due to the electron pool in the source is neglected, which, as shown below, is not justified for high electron densities.

\subsubsection{Short Channels}

The short channels studied here had a shape as depicted in Fig. 1c. They resemble more a point contact, with an effective length on the order of the width of $10 \mu \mathrm{m}$.

An example for the electron transport through such a point contact is shown in Fig. 5. As before, for each data point the source was filled to the same level, $10^{9}$ electrons in this case.

We again concentrate on the initial phase after opening the gate, when only a few percent of the electrons in the source are leaving through the channel. The data indicate once more that in this range the number of electrons transmitted through the channel during one pulse increases linearly with pulse width. The slope of the straight line is $\mathrm{d} N / \mathrm{d} t=2.6 \times 10^{12} \mathrm{e} / \mathrm{s}$, close to the long channel in Fig. 4 .

The comparable value for a long and a small channel of similar cross section could be a coincidence, but still it appears surprising at first sight, because the resistance of a point contact is expected to be much smaller than that of a long wire or channel. One has to keep in mind, however, that here we do not apply a certain voltage to the ends of the channel (as is usual for an electrical resistor), but the electrons in the channel are exposed to a certain external driving field $(0.5 \mathrm{~V} / \mathrm{cm}$ in Figs. 4, 5), which acts on each individual electron. The velocity of the electrons in the channel and hence the current should therefore be the same for a short and a long channel under otherwise identical conditions.

A similar consideration as in the previous section allows one to get an estimate for the upper limit of the mobility for this point contact: For a current of $2.6 \times 10^{12} \mathrm{e} / \mathrm{s}$, an electron density of $10^{10} \mathrm{~cm}^{2}$, an effective width of $10 \mu \mathrm{m}$, and an applied electric field of $0.5 \mathrm{~V} / \mathrm{cm}$, we find $\mu=5.5 \times 10^{5} \mathrm{~cm}^{2} / \mathrm{Vs}$.

\subsection{Multiple Pulses}

In the measurements to be described now the gate was opened not only once, but by a sequence of multiple pulses, without recharging the source (Fig. 6). The total number of 

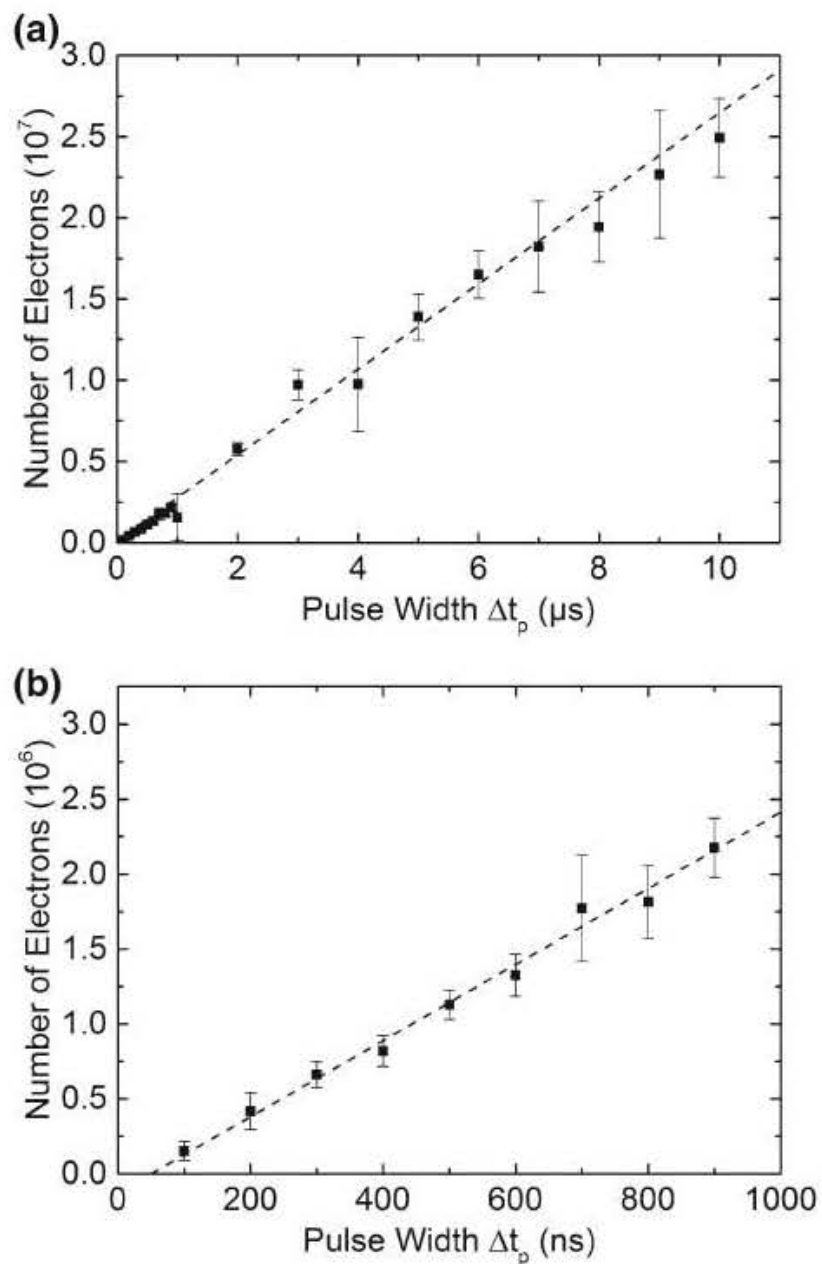

Fig.5 a Number of electrons transmitted from source to drain as the gate is opened for times short compared to the depletion time. The total number of electrons in the source was here $N_{s} \sim 10^{9}$. b Same as in (a) for the regime of $\mathrm{ns}$ pulses. The shape of the gate channel in this sample was like a point contact (geometrical width $10 \mu \mathrm{m}$ ); therefore, the channel length was not well defined, but effectively was comparable to the width. The slope of the straight lines corresponds to a current of $2.6 \times 10^{12} \mathrm{e} / \mathrm{s}$ (from Ref. [14])

electrons transmitted for a certain number of pulses in the burst was recorded, and then the source was again filled to saturation. Thereafter, the next data point with a different number of pulses was taken. The result is shown in Fig. 6 for up to $10^{4}$ pulses per burst and for three different pulse lengths $\Delta t_{\mathrm{p}}$. The sample used for this measurement was a long structured channel (like in Fig. 1d) with dimensions $3.75 \mu \mathrm{m} \times 400 \mu \mathrm{m}$.

It is observed that after a few hundred pulses, the total amount of electrons removed from the source saturates. The saturation level is different for the different pulse width, which implies that even after $10^{4}$ pulses there are still electrons left in the source for the runs with pulse widths $\Delta t_{\mathrm{p}}=0.5$ and $2 \mu \mathrm{s}$. For $10-\mu$ s pulses, the saturation level 


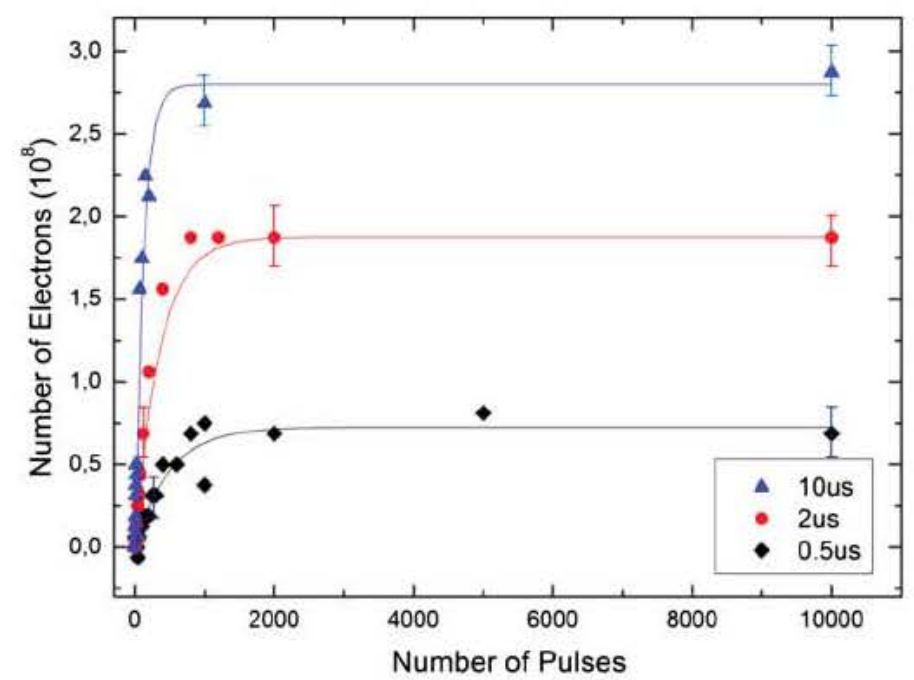

Fig. 6 Number of electrons transmitted from source to drain as a function of the number of applied gate pulses. The width of the pulses $\Delta t_{\mathrm{p}}$ in the three runs was $0.5,2.0$, and $10 \mu \mathrm{s}$, respectively. For each data point, the source was charged to $2.8 \times 10^{8}$ electrons before the measurement. A few representative error bars are shown. The channel in this sample was $400 \mu \mathrm{m}$ long and structured like in Fig. 1d (Color figure online)

is equal to the total number of electrons in the source; hence, only in this case the source is completely emptied by the pulse sequence.

The different saturation levels are obviously caused by the drainage of the source. We interpret this observation as a signature for the contribution of the electric field of the electrons in the source to the electron transport: If only the externally applied potential $V_{\text {sd }}$ were responsible for the electron current through the channel, the source should be emptied completely also by short pulses, if the pulse sequence is long enough. However, if the mutual repulsion of the electrons contributes to the transport, then this part drops as the source is gradually emptied and therefore the charge density at the channel entrance decreases. Eventually, the force due to the externally applied field will not be large enough for the electrons to reach the middle of the gate channel while the gate is open. These electrons will be pushed back into the source as soon as the gate barrier is switched on again at the end of the opening pulse, and they will thus not reach the drain.

\subsection{Quasi-dc Measurements}

In order to check for consistency, measurements on the same sample as in Fig. 5 (short channel) were taken by a complementary method: While in the experiments described so far the source was always filled up to saturation before a data point was taken and then the filament was switched off, in this run the source was charged continuously by the filament and the gate was opened repetitively for a certain pulse duration $\Delta t_{\mathrm{p}}$. Figure 7 shows an example for a repetition rate of $1000 \mathrm{~s}^{-1}$. 


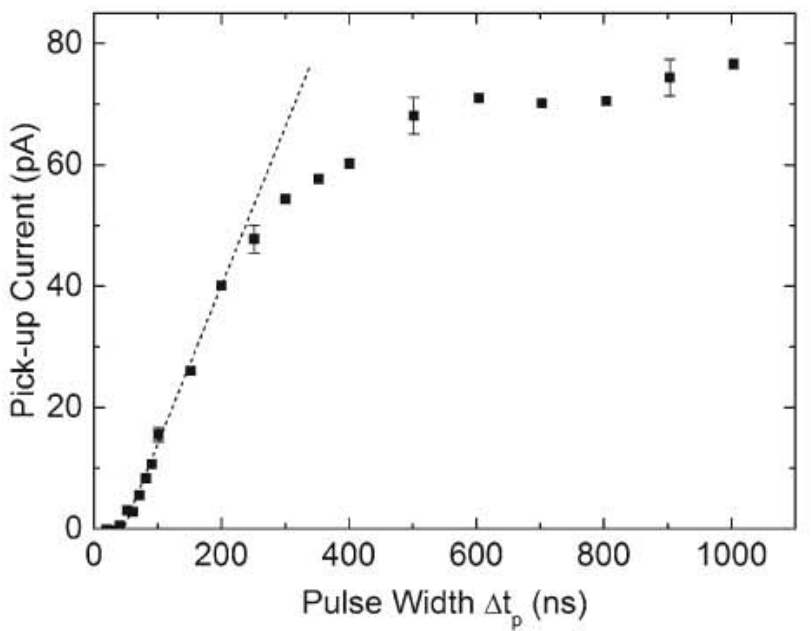

Fig. 7 Average current through a 'point contact' (same sample as in Fig. 5) that is opened periodically at a rate of $1000 \mathrm{~s}^{-1}$ by a gating pulse of duration $\Delta t_{\mathrm{p}}$

As the width of the gating pulses is increased, the pickup current initially displays a similar linear increase as it is seen in the previous figures for the transmitted charge of individual pulses. The saturation which in Fig. 7 sets in around $500 \mathrm{~ns}$ is here not limited by the total charge in the source, but by the finite supply current of the filament. This current can be determined when the gate is continuously open: The maximum current picked up at the drain was then $75 \mathrm{pA}$, which agrees with the saturation value in Fig. 7. It should be mentioned that the separation between subsequent pulses, $1 \mathrm{~ms}$, was very large compared to the time interval shown in Fig. 7, meaning that the source for all the data points in this plot was always charged to the same value.

We can determine the number of source electrons in this run from the following consideration: The average maximum pickup current of $75 \mathrm{pA}$ corresponds to a charge of $0.46 \times 10^{6}$ electrons transported per pulse at a rate of 1000 pulses per second. From the slope of the initial linear portion in Fig. 7, $25 \mathrm{pA} / 100 \mathrm{~ns}$, we get that a charge of $25 \mathrm{pC} / 1000$ or $1.6 \times 10^{5}$ electrons are transported per $100 \mathrm{~ns}$.

This current of $1.6 \times 10^{12} \mathrm{e} / \mathrm{s}$ is of the same order, but somewhat less than the value of $2.6 \times 10^{12} \mathrm{e} / \mathrm{s}$ obtained from the data shown in Fig. 5 for single pulses through the same sample. The difference might arise from some sample degradation, e.g., from some increased surface roughness, because the data in Fig. 7 were taken a few weeks after those in Fig. 5. However, of influence might also be the fact that the total charge in the source was only $0.46 \times 10^{6}$ electrons and hence the charge density at the channel entrance was here distinctly smaller than in Fig. 5. This is in line with the proposition that the Coulomb repulsion of the SSE in the source contributes to the electron transport in our device: For a smaller charge density, this contribution will be reduced, leading to a smaller current.

The data in Fig. 7 display an obvious delay in the onset of the transport current. Only for pulses longer than $50 \mathrm{~ns}$, charge transport could be detected. An indication for the same behavior, yet not as clear, can also be derived from the data in Fig. 5b. This 
delayed onset was interpreted in a previous publication as a signature for the finite transit time of the SSE through the gate channel [14]. However, another source for a delayed onset also has to be considered: The finite resistance of the electrical leads in the cryostat $(30 \Omega)$ and the gate capacitance $(3 \mathrm{nF})$ give an $R C$ of this circuit of $10^{-7} \mathrm{~s}$. As a result, the originally rectangular gating pulses arrive somewhat distorted at the gate channel and for pulses shorter than $\sim 100 \mathrm{~ns}$, open the channel for times distinctly less than the nominal pulse width. This generates the major part of the observed offset of $50 \mathrm{~ns}$. The true transit time of the electrons through the contact therefore has to be shorter than $50 \mathrm{~ns}$.

From this fact one can obtain a lower bound for the electron mobility in the channel: At an effective length of the constriction of $\sim 10 \mu \mathrm{m}$, the electron velocity $v$ has to be higher than $100 \mathrm{~m} / \mathrm{s}$ in order for an electron to reach the contact center in less than $50 \mathrm{~ns}$. This implies an electron mobility $\mu=v / E>2 \times 10^{4} \mathrm{~cm}^{2} / \mathrm{Vs}$, if we only take the external electric field $E=0.5 \mathrm{~V} / \mathrm{cm}$ into account. With the contribution of the Coulomb field of the source electrons, this estimate has to be reduced accordingly. This lower-bound estimate shows, nevertheless, that the mobility of SSE in microscopic structures can be quite high.

\subsection{Simulations}

As shown in Ref. [13] and already mentioned in the beginning, the charge density in the source is not constant across the source area. When the gate is closed and the current flow thus blocked, the electrons arrange under the influence of the externally applied potential $V_{\text {sd }}$ in the substrate in such a way that the chemical potential is constant throughout the charge pool in S. Because of the potential gradient in the substrate, this implies that the charge density is highest directly in front of the gate. As pointed out before, when the gate is opened and a current starts to flow, the electrons in front will not only be set in motion by the externally applied potential gradient stemming from the substrate, but also by the Coulomb repulsion of the charge in the source. The electrons will therefore move faster than they would solely under the effect of the external field on the order of $1 \mathrm{~V} / \mathrm{cm}$ applied along the channel. This modifies the estimates on the electron mobility given above.

In order to qualitatively illustrate the behavior of the electron pulse under such conditions, we have carried out a numerical simulation. Here we give only a brief description of the main results; a more complete treatment will be the subject of a separate paper. In short, we have used Brownian dynamics of particles under the influence of an external potential gradient and with repulsive particle-particle interaction [19]. The simulation was greatly simplified by calculating the diffusion trajectories of only 12 particles in the channel. Instead of the repulsive Coulomb interaction of our experimental system (which is modified by image charges in the substrate and hence contains dipolar contributions $[18,20]$ ), we have used in the calculations a pure repulsive dipole-dipole interaction, which varies with particle distance $r$ as $1 / r^{3}$. The simulation was originally devised to model the channel transport of $2 \mathrm{D}$ ensembles of paramagnetic colloidal particles, which are also interacting via dipolar forces. A description of these systems and the calculations can be found in Refs. [19,21]. The 


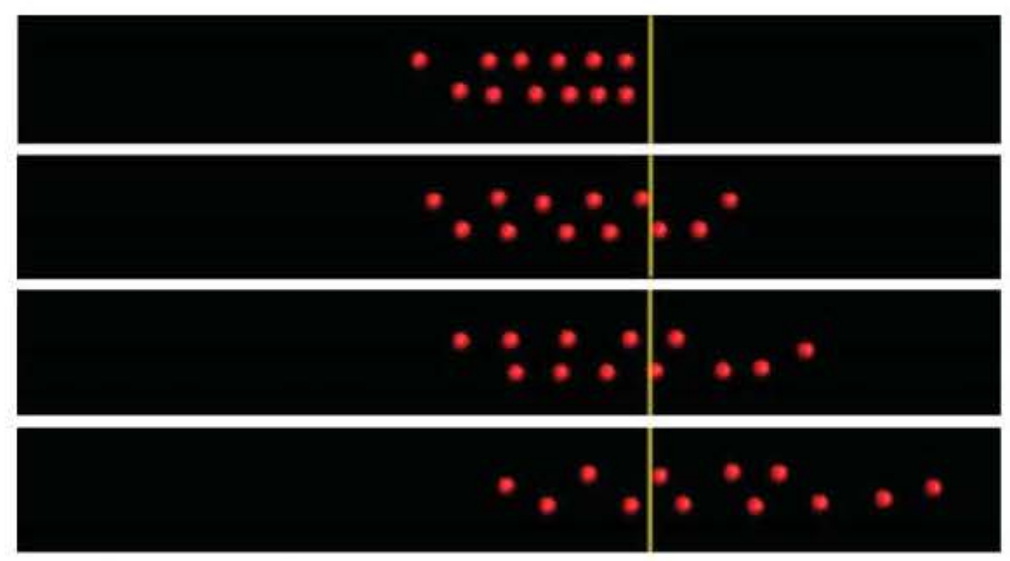

Fig. 8 Snapshots of particle configurations after different time steps, corresponding to the trajectories in Fig. 9. a $t=76, \mathrm{~b} t=342, \mathbf{c} t=402$, and $\mathbf{d} t=520$. The barrier is switched off at $t=300$, and the position of the barrier is indicated by the yellow line (Color figure online)

simulation was carried out for a temperature $T=295 \mathrm{~K}$ and hence a thermal energy per particle $E_{\text {th }}=4.1 \times 10^{-21} \mathrm{~J}$, an external force of $4.8 \times 10^{-15} \mathrm{~N}$ due to a (gravitational) potential gradient, and an interparticle force of $4.2 \times 10^{-14} \mathrm{~N}$ at an average particle distance of $20 \mu \mathrm{m}$. This leads to a potential energy $E_{\mathrm{pot}}=3.75 \times 10^{-20} \mathrm{~J}$ and a plasma parameter $\Gamma=E_{\text {pot }} / E_{\text {th }}$ of about 10 . Although the details of this colloidal system and the SSE are quite different, the relevant quantities are comparable: Both the ratio between the interparticle repulsive force and the force due to the external potential gradient on the one hand and the plasma parameter $\Gamma=E_{\mathrm{pot}} / E_{\mathrm{th}}$ on the other are on the order of 10 . One can expect, therefore, that effects of the interparticle repulsion on the particle transport are qualitatively reproduced by the simulation.

The procedure in the simulation was similar to our experiment with SSE: The channel was first blocked by a barrier, until the stored particles had reached thermodynamic equilibrium. Then the barrier was instantaneously switched off, and the drift of the particles under the influence of the potential gradient was recorded.

Figure 8 shows the snapshots of the particle configurations at different instances: Fig. 8a shows the equilibrium configuration before the barrier is turned off. The particle-particle distance is governed by the mutual repulsion of the particles and the external potential gradient along the channel. As in the experimental SSE system, the particle density increases for decreasing distance from the barrier [13]. This compression in front of the barrier follows from the pressure exerted by the particles further away from the barrier. For the choice of parameters used here, the particles form two lanes. This laning is well known not only for colloidal particle ensembles [21,22], but for SSE in a channel [7].

Figure $8 \mathrm{~b}$ represents the situation shortly after the barrier has been removed. Three particles have already passed the former position of the barrier, and the whole particle ensemble has expanded, exhibiting now a zigzag-like configuration, as calculated already by Peeters for Coulomb systems in a channel [23]. This expansion, mediated by particle-particle repulsion, continues in Fig. 8c, d, until eventually a situation 

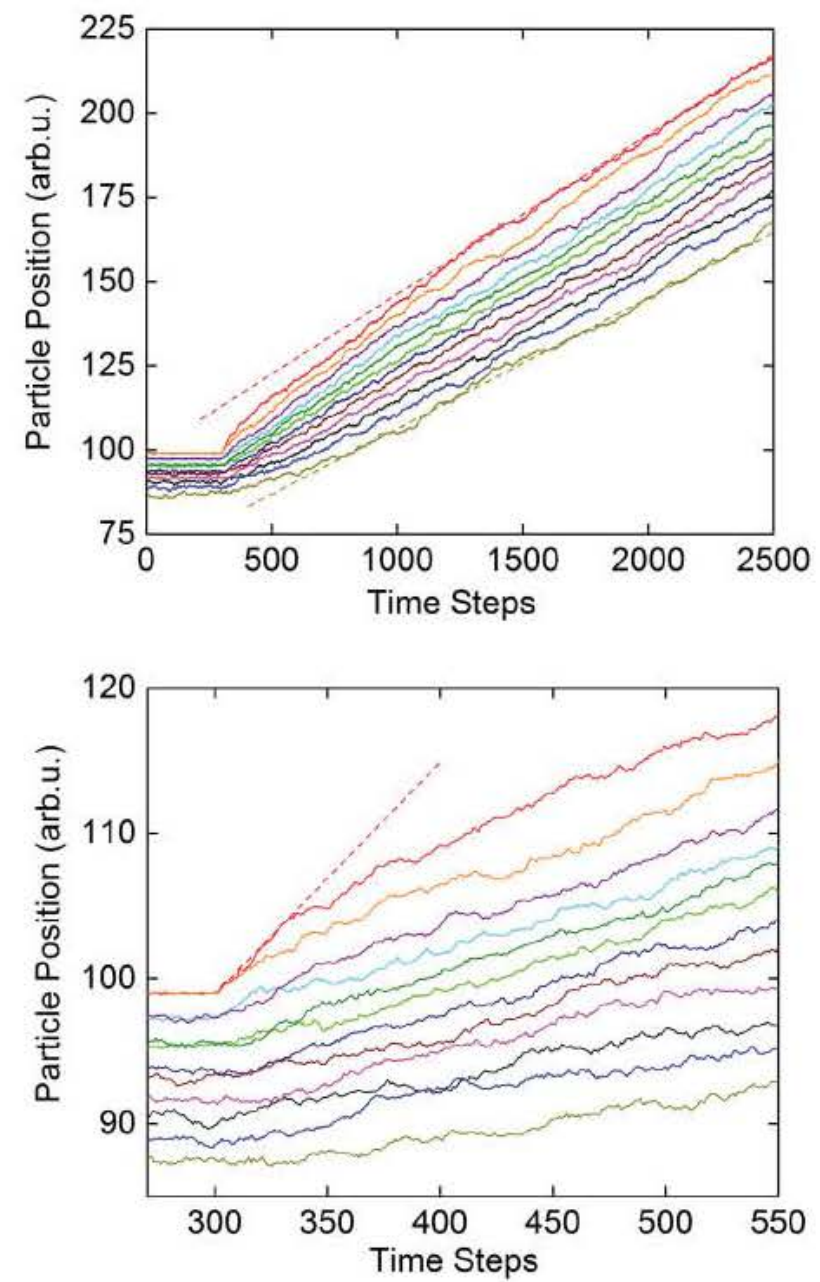

Fig. 9 (Top) trajectories of 12 repulsively interacting particles in a channel, exposed to an external potential gradient. The trajectories were obtained from a Brownian dynamics simulation. In the beginning, the particles are blocked by a barrier in the channel. At $t=300$, the barrier is removed and the particles drift along the channel under the influence of the external potential gradient. The slopes of the two dashed lines show that the velocities of the first and the last particle (marked red and green, respectively) are approaching each other, as time proceeds, but even after 2500 time steps still differ by $10 \%$. (Bottom) blowup of the initial phase after the barrier is switched off. In the beginning, the velocity of the first particle, as derived from the dashed line, is a factor of 4 higher than the average velocity of the ensemble after 2500 time steps (Color figure online)

is reached (not shown here) where the particle distance is large and the repulsive potential hence weak enough that it can be neglected compared to the thermal energy. What is also obvious from Fig. $8 \mathrm{c}, \mathrm{d}$ is that the particles tend to form only one lane in the channel as the interparticle distance increases. Such a behavior is expected when the interparticle distance is of the order of the effective channel width or larger. Experimentally, this can be realized by gradually varying the gate potential [7], even 
if the geometrical width of the gate channel, given by the distance of the split-gate electrodes, is large compared to the particle distance. In our SSE experiment, however, the electron-electron distance was in most cases distinctly smaller than the channel width; the laning effect should therefore play only a minor role in the experimental SSE data presented above.

The effect of repulsion on the particle transport, on the other hand, should be quite similar - at least qualitatively_-for the SSE experiment and the simulation. The spreading out of the particle cloud in Fig. 8 implies, apparently, that the front particles move at a speed higher than the average drift velocity, which is governed by the external potential gradient. This is exemplified more clearly in Fig. 9, which shows the positions of the 12 particles along the channel as a function of time. Before the barrier is opened, the particles close to it are more or less fixed in their positions due to the pressure of the particles further back. For the last particles, in contrast, Brownian motion leads to large fluctuations in their positions. As soon as the barrier is switched off, the particles start drifting downstream. The particle velocities in this plot are proportional to the slope of the individual trajectories. It is obvious that the velocities of the front particles (red and orange) are largest and that the whole ensemble spreads out. This clearly demonstrates the influence of interparticle interaction on the transport under the conditions envisaged here.

\section{Conclusions}

The results presented in this work demonstrate that quantitative studies of helium film SSE transport through micro-constrictions are feasible, in spite of complications like surface roughness and trapped charges that appear when the SSE are supported not by a thick, but only by a thin helium film. In contrast to previous transport studies, the measurements here were taken under conditions far from equilibrium, and clear indications for the effect of the Coulomb field of the electrons on the transport were found. On the basis of the reported results, it will now be possible to carry out systematic investigations on the influence of relevant parameters such as charge density, voltage bias. So far, the SSE transport in gated channels could be controlled on a timescale in the 100-ns regime, mainly limited by deficiencies of the gate circuitry, and much faster manipulation should be possible with an improved design. A major goal for future experiments will be transport studies of SSE in other regions of the phase diagram, e.g., in the Wigner crystal regime, where experiments close to equilibrium are already available [8] and where new phenomena could arise under strongly non-equilibrium conditions. Particularly interesting will be the transport behavior at high electron densities near the quantum melting of the Wigner crystal [24]. Moreover, transport through more complex microchannels appears in reach, in order to study electron tunneling across potential barriers, Coulomb blockade, and transport through very narrow constrictions comparable to the mean free path of the SSE. The silicon substrates used here appear also interesting for other investigations of SSE systems on thin films, especially at high frequencies [20], because the conductivity of this material can be tuned in situ over a wide range by external illumination. 
Acknowledgments It is a pleasure to dedicate this work to Horst Meyer on the occasion of his 90th birthday. One of us (P.L.) has the privilege of knowing Horst for more than four decades and has profited tremendously from many illuminating discussions on various aspects of quantum fluids and solids. We thank V. B. Shikin for useful correspondence and Louis Kukk for technical help. M. A. gratefully acknowledges the support by DAAD, and F. S. a scholarship by the University of Khartoum.

\section{References}

1. E. Andrei, Two-Dimensional Electron Systems on Helium and Other Cryogenic Substrates (Kluwer Academic, Dordrecht, 1997)

2. C.C. Grimes, G. Adams, Phys. Rev. Lett. 36, 145 (1976)

3. K. Shirahama, S. Ito, H. Suto, K. Kono, J. Low Temp. Phys. 101, 433 (1995)

4. P. Leiderer, E. Scheer, K. Kono, J.-J. Lin, D.G. Rees, J. Low Temp. Phys. (2015). doi:10.1007/ s10909-015-1377-8

5. P.M. Platzman, M.I. Dykman, Science 284, 5422 (1999)

6. D.I. Schuster, A. Fragner, M.I. Dykman, S.A. Lyon, R.J. Schoelkopf, Phys. Rev. Lett. 105, 40503 (2010)

7. D.G. Rees, I. Kuroda, C.A. Marrache-Kikuchi, M. Höfer, P. Leiderer, K. Kono, Phys. Rev. Lett. 106, 026803 (2011)

8. H. Ikegami, H. Akimoto, D.G. Rees, K. Kono, Phys. Rev. Lett. 109, 236802 (2012)

9. M. Takita, C.S. Nichols, S. Lyon, Bull. Am. Phys. Soc. 60(1), (2015). http://meetings.aps.org/link/ BAPS.2015.MAR.Q37.11

10. P. Glasson, V. Dotsenko, P. Fozooni, M.J. Lea, W. Bailey, G. Papageorgiou, S.E. Andresen, A. Kristensen, Phys. Rev. Lett. 87, 176802 (2001)

11. H. Ikegami, H. Akimoto, K. Kono, Phys. Rev. Lett. 102, 046807 (2009)

12. J. Klier, I. Doicescu, P. Leiderer, J. Low Temp. Phys. 121, 603 (2000)

13. M. Ashari, D.G. Rees, K. Kono, E. Scheer, P. Leiderer, J. Low Temp. Phys. 167, 15 (2012)

14. F. Shaban, T. Lorenz, R. Rau, M. Ashari, D. Rees, K. Kono, E. Scheer, P. Leiderer, J. Phys. Conf. Ser. 568, $012008(2014)$

15. V. Shikin, J. Klier, I. Doicescu, A. Würl, P. Leiderer, Phys. Rev. B 64, 073401 (2001)

16. X.L. Hu, Y. Carmi, A.J. Dahm, J. Low Temp. Phys. 89, 625 (1992)

17. E.Y. Andrei, Phys. Rev. Lett. 52, 1449 (1984)

18. H.W. Jiang, M.A. Stan, A.J. Dahm, Surf. Sci. 196, 1 (1988)

19. C. Kreuter, U. Siems, P. Henseler, P. Nielaba, P. Leiderer, A. Erbe, J. Phys. Condens. Matter 24, 464120 (2012)

20. G. Mistura, T. Günzler, S. Neser, P. Leiderer, Phys. Rev. B 56, 8360 (1997)

21. M. Köppl, P. Henseler, A. Erbe, P. Nielaba, P. Leiderer, Phys. Rev. Lett. 97, 208302 (2006)

22. U. Siems, C. Kreuter, A. Erbe, N. Schwierz, S. Sengupty, P. Leiderer, P. Nielaba, Sci. Rep. 2, 1015 (2013)

23. G. Piacente, I.V. Schweigert, J.J. Betouras, F.M. Peeters, Phys. Rev. B 69, 045324 (2004)

24. T. Günzler, B. Bitnar, G. Mistura, S. Neser, P. Leiderer, Surf. Sci. 361/362, 831 (1996) 